\title{
Treinamento físico intervalado como ferramenta na terapia cognitivo- comportamental do transtorno de pânico
}

\author{
Interval aerobic training as a tool in the cognitive- \\ behavioral treatment of panic disorder
}

Aline Sardinha', Claudio Gil Soares de Araújo², Antonio Egidio Nardi²

\section{RESUMO}

Este trabalho consiste no relato da utilização de um programa de exercícios físicos aeróbicos intervalados no contexto da terapia cognitivo-comportamental em um caso de transtorno de pânico e agorafobia. São descritos os procedimentos utilizados, bem como os resultados positivos obtidos na redução da ansiedade cardíaca e na ansiedade cotidiana em relação a situações que exigem esforço físico.

\begin{abstract}
The present article reports the use of an interval aerobic exercise program as an adjunct intervention in the context of cognitive behavioural therapy in a patient with panic disorder and agoraphobia. We describe the procedures used in the exercise program and also the positive results achieved regarding cardiac anxiety reduction and everyday anxiety reduction concerning activities that requested physical effort.
\end{abstract}

\section{INTRODUÇÃO}

Indivíduos com transtorno de pânico (TP) experimentam diferentes manifestações de natureza autonômica durante os ataques, sendo possível identificar alguns subtipos distintos de apresentação do quadro'. Uma característica comum a todos os subtipos, entretanto, é a ansiedade relacionada à ocorrência de sintomas de pânico e a estímulos percebidos como ameaçadores à saúde. A ansiedade relacionada à saúde pode ser considerada, assim, um fator constituinte da fisiopatologia do TP².

A ansiedade cardíaca é um tipo de ansiedade relacionada à saúde em que as preocupações do paciente estão especificamente voltadas para o sistema cardiovascular e para a possibilidade de ter um evento cardiovascular agudo ou de desenvolver uma doença coronariana ${ }^{3}$. Essa envolve ainda dois outros fatores: comportamentos de evitação de atividades ou exercícios físicos e situações desencadeantes de sintomas cardiovasculares percebidos como perigosos e a hipervigilância para a ocorrência de tais sintomas4. Em uma revisão recente ${ }^{5}$, pudemos evidenciar que os exercícios físicos podem desempenhar um papel importante no tratamento do TP; isso se manifesta primariamente por meio de seu efeito antipânico agudo ${ }^{6}$ e na melhora dos sintomas com a prática regular? Algumas hipóteses mecanísticas foram aventadas para explicar tal efeito, incluindo o aumento dos níveis de fator neurotrófico cerebral $(B D N F)^{8}$ e o sistema serotoninérgico9. É possível ainda que, dentro da hipótese do condicionamento interoceptivo ${ }^{10}$, a exposição gradual ao exercício físico possa promover habituação do sistema

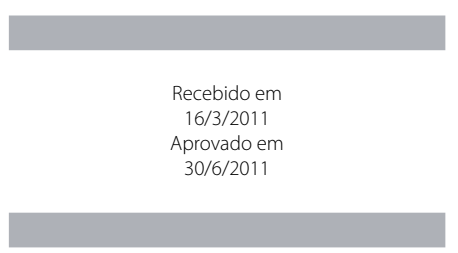

1 Universidade Federal do Rio de Janeiro (UFRJ), Instituto de Psiquiatria, Laboratório de Pânico e Respiração, Programa de Pós-graduação em Psiquiatria e Saúde Mental; INCT - Translational Medicine (CNPq).

2 Clínica de Medicina do Exercício - Clinimex; Universidade Gama Filho (UGF), Programa de Pós-graduação em Educação Física.

Endereço para correspondência: Aline Sardinha

Rua Visconde de Pirajá, 142/1405 - Ipanema - 22410-000 - Rio de Janeiro, RJ

E-mail: alinesardinhapsi@gmail.com 
do medo às alterações da frequência cardíaca $(F C)$, especialmente naqueles pacientes com maiores escores de ansiedade cardíaca. Nesse sentido, uma das modalidades mais clássicas de treinamento físico - treinamento intervalado -, ao proporcionar ciclos de períodos de intensidade variável de exercício"1 ${ }^{11}$, poderia ser especialmente conveniente.

Não foi encontrado, contudo, na literatura, nenhum estudo envolvendo esse tipo de treinamento em pacientes com TP. Sendo assim, o objetivo do presente relato de caso foi ilustrar a aplicação do treinamento intervalado supervisionado como ferramenta na terapia cognitivo-comportamental (TCC) no TP e discutir seu potencial de eficácia e aplicabilidade.

\section{DESCRIÇÃO DO CASO}

Paciente feminina de 52 anos relata ataques de pânico desde os 17 anos, iniciados após um período de longa enfermidade seguido de falecimento de sua mãe. A história familiar é positiva para TP por parte de um irmão. Não há registro de outros casos de transtornos psiquiátricos na família. Ao longo de todos esses anos, a paciente experimentava sintomas predominantemente cardiovasculares e respiratórios, como taquicardia, dispneia, aperto no peito e tontura, acompanhados de agorafobia. Nesse período, a paciente submeteu-se a diversos tratamentos psicodinâmicos e medicamentosos com antidepressivos tricíclicos, benzodiazepínicos e, nos últimos 24 meses, com uso de escitalopram e alprazolam. Nesses 35 anos de evolução do TP, a paciente alternou momentos de remissão com outros mais graves, com ataques frequentes e significativa perda de qualidade de vida. A agorafobia permaneceu ao longo do tempo. Em decorrência das queixas cardiovasculares, a paciente foi por diversas vezes submetida a investigações cardiovasculares, tendo apenas sido diagnosticado um prolapso da válvula mitral sem insuficiência importante ou degeneração mixomatosa; o sintoma de taquicardia chegou a ser tratado, por aproximadamente 1 ano, com betabloqueador. A paciente apresenta comorbidade com transtorno da ansiedade generalizada (TAG) e transtorno do déficit de atenção e hiperatividade (TDAH). Seus dois filhos têm diagnóstico de TDAH.

Há 2 anos foi encaminhada para a TCC, obtendo a remissão dos ataques de pânico e melhora significativa da agorafobia, ganhando mobilidade e podendo retomar suas atividades profissionais. Nesse momento, seu escore no Clinical Global Impression Scale (CGI) era 5. As intervenções cognitivo-comportamentais utilizadas para o tratamento do pânico consistiram predominantemente em reestruturação cognitiva, psicoeducação sobre os sintomas de ansiedade, técnicas de relaxamento e respiração diafragmática, exposições imaginárias, exposições ao vivo em situações evitadas e exposições interoceptivas. Muitos ganhos terapêuticos foram obtidos com a TCC, alcançando melhora significati- va para o TAG e o TDAH, a remissão dos ataques de pânico e da evitação agorafóbica. Entretanto, mesmo após 2 anos de TCC, a paciente continuava a experimentar ansiedade e a evitar situações que pudessem desencadear taquicardia, como subir escadas, caminhar em ritmo normal ou praticar exercícios físicos $(C G I=3)$. Um aspecto interessante de seu relato é que a taquicardia ocorria ao final do exercício, quando terminava de subir as escadas ou quando parava de caminhar e não durante sua vigência. Essa inatividade física relativa foi também acompanhada de um expressivo ganho ponderal - cerca de $13 \mathrm{~kg}$ nos últimos 15 anos -, contribuindo para uma pior qualidade de vida.

Foi proposto à paciente, então, iniciar um programa de exercício físico supervisionado por um médico e uma psicoterapeuta, que funcionaria como exposição interoceptiva, no contexto da TCC. O primeiro passo consistiu na realização de uma avaliação médico-funcional completa, que incluiu um teste cardiopulmonar de exercício máximo em cicloergômetro com protocolo de rampa ${ }^{12}$ para avaliar seu estado de saúde e de aptidão física, permitindo obter dados objetivos que guiassem a prescrição do programa de exercício da paciente. Foi observado que a carga alcançada se encontrava em $40 \%$ da prevista para idade, gênero e peso corporal, com um consumo máximo de oxigênio de $15,8 \mathrm{~mL} / \mathrm{kg}^{-1} \cdot \mathrm{min}^{-1}$ ou 4,5 METs (equivalentes metabólicos), cerca de $55 \%$ do previsto, indicando um nível extremamente baixo de condicionamento físico, provavelmente decorrente da evitação sistemática de atividades físicas pela paciente. A FC máxima obtida no teste foi de 160 bpm. O tônus vagal cardíaco, estimado pelo teste de exercício de 4 segundos ${ }^{13}$, estava abaixo do percentil 5 para a faixa etária.

Em seguida, iniciou-se o treinamento aeróbico em esteira, realizado de maneira intervalada, alternando caminhadas lentas e mais rápidas a cada 2 minutos. Na tabela 1 é apresentado o detalhamento de 12 sessões de treinamento.

A ansiedade cardíaca foi quantificada antes e ao final do treinamento pela aplicação do Questionário de Ansiedade cardíaca (QAC), desenvolvido e validado por Eifert et al. ${ }^{4}$, por meio de sua versão traduzida e adaptada para a população brasileira por Sardinha et al. ${ }^{14}$. Nesse instrumento, o paciente informa sobre sua ansiedade cardíaca em uma escala de 18 itens com pontuações variando numa escala Likert entre 1 e 5. Sua estrutura fatorial inclui três subescalas: medo e preocupação com sensações cardíacas, evitação cardioprotetora de atividades que podem ocasionar sintomas, e atenção e monitoramento cardíaco de estímulos relacionados ao coração. A ansiedade antecipatória e durante o exercício foi medida pela Subjective Units of Distress Scale (SUDS), em que o paciente atribui um grau de 0 a 10 à ansiedade experimentada.

Após 12 sessões, pode-se observar uma redução da ansiedade durante o exercício, passando de valores entre 6 e 7 para apenas 2, e da ansiedade cardíaca, medida por meio do QAC, de 58 para 37 pontos. Em relação à ansiedade cardíaca 
Tabela 1. Protocolo do treinamento físico aeróbico intervalado utilizado

\begin{tabular}{|c|c|c|c|c|c|c|c|}
\hline $\begin{array}{l}\text { Número da sessäo de } \\
\text { exercício }\end{array}$ & $\begin{array}{l}\text { Velocidade mínima } \\
(\mathrm{km} / \mathrm{h})\end{array}$ & $\begin{array}{l}\text { Velocidade máxima } \\
\qquad(\mathrm{km} / \mathrm{h})\end{array}$ & $\begin{array}{c}\mathrm{FC} \text { (mínima } \\
(\mathrm{bpm})\end{array}$ & $\begin{array}{c}\text { FC máxima } \\
(\mathrm{bpm})\end{array}$ & $\begin{array}{c}\text { Escala de percepção de } \\
\text { esforço (0-10) }\end{array}$ & Ansiedade (0-10) & $\begin{array}{l}\text { Ansiedade cardíaca } \\
\text { (QAC) }\end{array}$ \\
\hline Pré-treinamento & & & & & & 7 & 58 \\
\hline 1 & 4,5 & 4,7 & 117 & 125 & $3-5$ & 6 & \\
\hline 2 & 4,5 & 4,7 & 105 & 119 & $3-3$ & 4 & \\
\hline 3 & 4,5 & 4,7 & 115 & 129 & $4-5$ & 3 & \\
\hline 4 & 4,5 & 5,2 & 118 & 144 & $3-6$ & 7 & \\
\hline 5 & 4,5 & 5,2 & 120 & 145 & $4-5$ & 6 & \\
\hline 6 & 4,5 & 4,8 & 115 & 137 & $3-3$ & 5 & \\
\hline 7 & 4,6 & 5,2 & 125 & 140 & $4-5$ & 5 & \\
\hline 8 & 4,6 & 5,2 & 117 & 125 & $5-5$ & 4 & \\
\hline 9 & 4,6 & 5,2 & 118 & 127 & $2-3$ & 3 & \\
\hline 10 & 4,8 & 5,4 & 119 & 135 & $3-3$ & 3 & \\
\hline 11 & 4,8 & 5,4 & 116 & 130 & $2-3$ & 2 & \\
\hline 12 & 4,8 & 5,4 & 113 & 134 & $1-3$ & 2 & 37 \\
\hline Média & & & 116 & 132 & & & \\
\hline $\begin{array}{l}\text { Percentual da FC } \\
\text { máxima* }\end{array}$ & & & $73 \%$ & $83 \%$ & & & \\
\hline
\end{tabular}

* Do valor obtido no teste cardiopulmonar de exercício máximo pré-início do programa de exercício supervisionado (160bpm).

FC: frequência cardíaca; QAC: Questionário de Ansiedade Cardíaca.

no cotidiano, a paciente relata melhoras na ansiedade percebida, que vão ao encontro dos escores mais baixos obtidos no QAC ao final das 12 sessões de treinamento físico. O escore do CGl atual da paciente é 1 .

Apesar de não ter sido realizado um novo teste cardiopulmonar de exercício máximo, é possível afirmar que houve uma melhora na condição aeróbica da paciente, uma vez que os escores de percepção de cansaço, medidos pela escala de Borg ${ }^{15}$, com valores entre 0 e 10 durante o exercício se reduziram e foi possível um aumento da velocidade e do tempo de caminhada rápida, assim como da carga no cicloergômetro, sem que houvesse um correspondente incremento da FC alcançada no exercício, o que é denominado de bradicardia submáxima relativa ao exercício e um dos maiores indicadores indiretos de ganho aeróbico ${ }^{16}$. Esse impacto tende a ser mais evidente logo nas primeiras sessões de treinamento e a magnitude da redução da FC máxima alcançada na caminhada a $5,4 \mathrm{~km} / \mathrm{h}$ reduziu-se em cerca de 10 a 15 batimentos por minuto (bpm).

A paciente continua frequentando o programa de exercício supervisionado três vezes por semana, agora já sem a supervisão obrigatória da terapeuta, em adendo às sessões semanais de TCC. A meta dessa intervenção conjunta é alcançar uma condição aeróbica e de estabilidade psicológica que a permita iniciar, gradativamente, a corrida em esteira. Em paralelo, nas sessões de TCC, vem sendo trabalhada a distinção entre episódios de taquicardia imotivados e o aumento da FC decorrente da resposta saudável e fisiológica do sistema cardiorrespiratório às demandas de uma determinada situação.

\section{DISCUSSÃO}

A utilização, neste caso, do treinamento físico intervalado dentro do contexto da TCC demonstrou eficácia na redução tanto da ansiedade relacionada ao exercício quanto da ansiedade cardíaca. De acordo com a hipótese do condicionamento interoceptivo como agente mantenedor do TP, nestes pacientes, o sistema do medo se encontra hiperreativo a estímulos interoceptivos decorrentes de alterações fisiológicas necessárias à manutenção da homestase do organismo ${ }^{10}$. Nesse sentido, as alterações autonômicas experimentadas durante a prática de exercícios físicos podem ser consideradas estímulos ansiogênicos - e até mesmo panicogênicos.

Por outro lado, o baixo condicionamento físico decorrente da evitação dessas situações é um fator potencialmente agravante do caso relatado, uma vez que a variabilidade da FC em pacientes sedentários, como era o caso, tende a ser maior ${ }^{17}$. Assim, uma paciente com um perfil sedentário e com níveis baixos de condição aeróbica, ao realizar um exercício físico de intensidade relativamente baixa em seu cotidiano, como subir um lance de escada, experimentava um aumento mais acentuado ou desproporcional da FC que, percebida por um sistema do medo hipersensível, desencadeava ansiedade e, consequentemente, mais sintomas autonômicos, podendo culminar em um ataque de pânico.

O treinamento aeróbico intervalar foi pensado como uma maneira de promover habituação da paciente a repetidas variações da FC, ao longo da sessão de exercício. Na medida em que, durante 30 minutos, a paciente foi exposta a aumentos e decréscimos em sua FC, foi possível promover 
habituação do sistema do medo, o que pode ser constatado pela redução da ansiedade percebida durante o exercício. Assim, apesar de não haver relatos na literatura da utilização de treinamento aeróbico intervalado na terapêutica do TP até o momento, é possível concluir, a partir desse caso, que a inserção de treinamento aeróbico intervalar em adição às medicações e à TCC pode ser uma estratégia terapêutica viável em casos de pacientes com TP e ansiedade cardíaca.

\section{AGRADECIMENTOS}

Aline Sardinha é bolsista de doutorado pelo Conselho Nacional de Desenvolvimento Científico e Tecnológico (CNPq). Claudio Gil Soares de Araújo e Antonio Egidio Nardi são bolsistas de produtividade do CNPq. Claudio Gil Soares de Araújo é ainda bolsista da Fundação de Amparo à Pesquisa do Estado do Rio de Janeiro (Faperj). Os autores agradecem à equipe da Clinimex - Clínica de Medicina do Exercício.

\section{REFERÊNCIAS}

1. Kircanski K, Craske MG, Epstein AM, Wittchen HU. Subtypes of panic attacks: a critical review of the empirical literature. Depress Anxiety. 2009;26(10):878-87.

2. Abramowitz JS, Olatunji BO, Deacon BJ. Health anxiety, hypochondriasis, and the anxiety disorders. Behav Ther. 2007;38(1):86-94.

3. Zvolensky MJ, Feldner MT, Eifert GH, Vujanovic AA, Solomon SE. Cardiophobia: a critical analysis. Transcult Psychiatry. 2008:45(2):230-52.

4. Eifert GH, Thompson RN, Zvolensky MJ, Edwards K, Frazer NL, Haddad JW, et al. The cardiac anxiety questionnaire: development and preliminary validity. Behav Res Ther. 2000;38(10):1039-53.
5. Sardinha A, Araújo CGS, Soares-Filho GL, Nardi AE. Treating the association of anxiety, panic disorder and coronary artery disease: issues concerning physical exercise and cognitive behavior therapy. Expert Rev Cardiovasc Ther. 2011;9(2):165-75.

6. Strohle A, Graetz B, Scheel M, Wittmann A, Feller C, Heinz A, et al. The acute antipanic and anxiolytic activity of aerobic exercise in patients with panic disorder and healthy control subjects. J Psychiatr Res. 2009;43(12):1013-7.

7. Broocks A, Bandelow B, Pekrun G, George A, Meyer T, Bartmann U, et al. Comparison of aerobic exercise, clomipramine, and placebo in the treatment of panic disorder. Am J Psychiatry. 1998;155(5):603-9.

8. Strohle A, Stoy M, Graetz B, Scheel M, Wittmann A, Gallinat J, et al. Acute exercise ameliorates reduced brain-derived neurotrophic factor in patients with panic disorder. Psychoneuroendocrinology. 2010;35(3):364-8.

9. Esquivel G, Diaz-Galvis J, Schruers K, Berlanga C, Lara-Munoz C, Griez E. Acute exercise reduces the effects of a 35\% CO2 challenge in patients with panic disorder. J Affect Disord. 2008;107(1-3):217-20.

10. Bouton ME, Mineka S, Barlow DH. A modern learning theory perspective on the etiology of panic disorder. Psychol Rev. 2001;108(1):4-32.

11. Araújo CGS, Carvalho T, Castro CL, Costa RV, Moraes RS, Oliveira-Filho JA, et al. Normatização dos equipamentos e técnicas da reabilitação cardiovascular supervisionada. Arq Bras Cardiol. 2004;83(5):448-52.

12. Meneghello RS, Araújo CGS, Stein R, Mastrocolla LE, Albuquerque PF, Serra SM. III Diretrizes da Sociedade Brasileira de Cardiologia sobre teste ergométrico. Arq Bras Cardiol. 2010;95(5 supl 1):1-26.

13. Araujo CGS, Castro CLB, Nóbrega ACL. Vagal activity: effect of age, sex and physical activity pattern. Braz J Med Biol Res. 1989;22(7):909-11.

14. Sardinha A, Nardi AE, Eifert GH. Tradução e adaptação transcultural da versão brasileira do Questionário de Ansiedade Cardíaca. Rev Psiquiatr RS. 2008;30(2):139-49.

15. Borg G. Borg's perceived exertion and pain scales. Champaign: Human Kinetics; 1998.

16. Almeida MB, Araújo CGS. Efeitos do treinamento aeróbico sobre a frequência cardíaca. Rev Bras Med Esporte. 2003;9(2):104-12.

17. Friedman BH. An autonomic flexibility-neurovisceral integration model of anxiety and cardiac vagal tone. Biol Psychol. 2007;74(2):185-99. 